\title{
Temporomandibular Joint Disorders- An Overview of Diagnosis and Treatment Options
}

\author{
Tofiq Hakimuddin Bohra ${ }^{1 *}$, Zainab T Bohra ${ }^{1}$ and Varun Kunte ${ }^{2}$ \\ ${ }^{1}$ Department of Oral and Maxillofacial Surgery, Apex Multispecialty Hospital, SRV \\ Group of Hospital, India \\ ${ }^{2} B d s$, Mds ,OMDR Specialist Tmj Disorders Director-Arthrose Craniofacial Pain \\ Clinic \\ *Corresponding Author: Tofiq Hakimuddin Bohra, Department of Oral and \\ Maxillofacial Surgery, Apex Multispecialty Hospital, SRV Group of Hospital, India.
}

Received: May 07, 2021

Published: June 03, 2021

(C) All rights are reserved by Tofiq

Hakimuddin Bohra., et al.

\begin{abstract}
Temporomandibular Joint (TMJ) Disorders is a lifestyle modulating disorder. Patients having this disorder go through a lot of pain, discomfort and restriction in the joint movement in day to day life. There are times when the patient is aware of the pain and discomfort but the diagnosis is not made on time, sometimes if the diagnosis is made the appropriate treatment is not started on time. These patients are passed on from doctors to doctors in search of an appropriate treatment. This article emphasizes on the need for the Dentist and Physician joining practice to understand the disorder along with the planning for the treatment. As TMJ disorders is a complex disorder requires right intervention in the right stage of the disorder. This article focuses on the various treatment options available till date for the management of TMJ disorders.
\end{abstract}

Keywords: Temporomandibular Disorders; TMJ; Orofacial Pain; Dental Occlusion; TMJ Arthroscopy

\section{Introduction}

Temporomandibular disorders (TMD) is a very broad term in clinical practice, it is a multifactorial disorder that may include joint, muscles and surrounding bony structures. Okeson (1996) described it as number of clinical problems that involve the muscles of mastication and the temporomandibular joint (TMJ), and its associated structures. Most patients with TMD come with a chief complaint of PAIN, followed by complaints such as joint noises, decreased range of mandibular motion, closed locked jaw, radiating pain over the jaw to the ears, headache etc. Women are considered to have a higher predilection towards TMD as compared to males, The reasons behind is the sexual disequilibrium in TMD, however the prevalence is not entirely clear but some have suggested a hormonal influence i.e. the level of estrogen hormone is seen higher in TMD patients. In fact, both animal and human studies have sug- gested that sex hormones may predispose to TMJ dysfunction and cartilaginous breakdown.

TMD is considered as a sequel of a multifactorial process like parafunctional habits, hyperfunction of group of masticatory muscles, previous trauma and articular changes of the joint. Historically, the role occlusion plays in TMD has proven controversial and inconclusive. Some studies demonstrate an association between TMD and occlusion (Pullinger., et al. J Prosthet Dent 59:363, 1988) and others find no connection (Dworkin., et al. J Am Dent Assoc $120: 273,1990)$. Given this lack of consensus and clear causal relationship between occlusion and TMD, the idea of treating TMD by making modifications to the occlusion seems unmerited. On the other hand, as a major component of the stomatognathic complex, the occlusion is of course an important consideration when formu- 
lating a diagnosis and treatment plan for the TMD patient. Specifically, it is critical to develop a stable and reproducible occlusion to achieve a stable result of various surgical and nonsurgical manipulations aimed at directly treating the TMD.

\section{Anatomy of the joint}

Temporomandibular Joint (TMJ) is ginglymoarthroidal joint that means it has a hinge as well as a sliding motion. TMJ is a complex joint with a number of movements like lateral, hinge, translation, slight rotational. It is composed of a condylar head of the mandible that articulates with the temporal bone of the skull in the fossa called as the Glenoid Fossa, restricted anteriorly by a prominence called as the articular eminence and posteriorly by the external auditory meatus i.e. a bony auditory canal. The joint also has an anatomical structure called as the articular disc or meniscus; it is a structure present between the mandibular condylar head and the glenoid fossa. In general terms you can imagine it as a structure placed between two bony surfaces that acts as a shock absorber and facilitates unhindered movement of the boy surfaces without interference.

This interposed disc is divided into three zones Anterior, Intermediate and Posterior, anteriorly this disc attaches to the lateral pterygoid muscle and posteriorly it attaches to the capsule by a bilaminar zone. Bilaminar zone also called as the Retrodiscal tissue is the part of the joint that has Blood vessels and Nerve innervation as compared to the articular disc that is avascular in nature.

The movement of the joint is divided in two parts: 1) Rotation of the condyle along its own axis that provides approximately 20 mm of mouth opening. 2) translation/Sliding of the joint along the articular eminence that provides the rest of the complete mouth opening. These movements are controlled by masticatory muscles of the joint, Temporalis, Masseter, lateral pterygoid and Medial pterygoid muscle. These muscles work in tandem inside a fibrous capsule of the joint thickened by the lateral (temporomandibular) ligament, which reinforces the lateral part of the capsule. The joint is also supported by the stylomandibular and sphenomandibular ligaments (Figure 1).

\section{Classification and diagnosis}

Most of the TMD can be classified as extracapsular and Intra capsular disorders, i.e. articular and non-articular disorders the

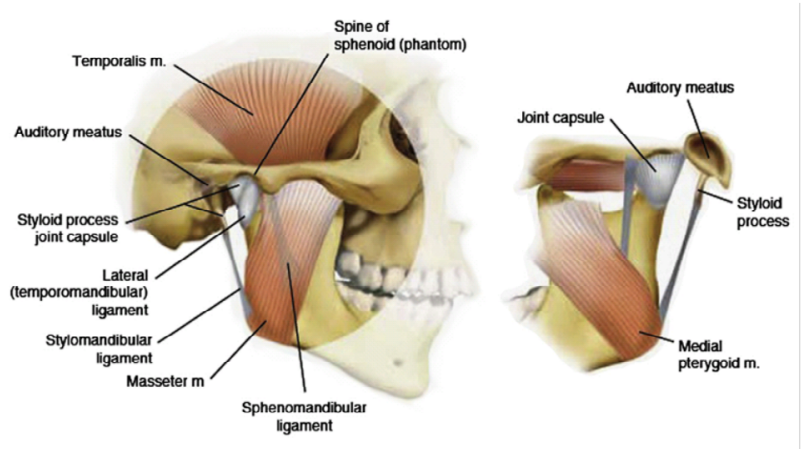

1. Musculoskeletal structures of TMJ (lateral and medial views).

Figure 1

extracapsular disorders refer to the pain that is caused in the Masticatory muscles surrounding the joint as a result of muscle load, spasm, myopathies. Whereas the intracapsular (articular) disorders refer to the discrepancies in the joint termed as 'Internal Derangement' i.e. it can be divided into inflammatory and non-inflammatory disorders, inflammatory disorders include Rheumatoid arthritis and Infective arthritis. Non inflammatory includes change in relationship between the disc and condyle i.e. disc displacement, osteoarthritis, joint damage due to trauma or surgery, mechanical trauma, cartilage or bone disease. Mechanically, articular disorders occur as a result of an altered balance of anabolic and catabolic cytokines. This cytokine imbalance creates an inflammatory milieu, which leads to oxidative stress, free radicals and ultimately joint damage (TMJ LUI). Apart from this classification Wilke's classification (Figure 2) is used to classify TMJ disorders.

Internal Derangement is a condition where the relationship between the condyle and the disc changes i.e. the disc is displaced with reduction or without reduction. In majority the disc is displaced anteriorly as compared to medially, laterally or posteriorly. Disc displacement with reduction is a condition where the disc is displaced and acts as an interference between the condyle and the articular eminence, when the disc returns back to its original position during opening and closing of mouth this condition is called as disc displacement with reduction and this is the point where the patient experiences a click, pop or crepitus sound. Disc displace- 


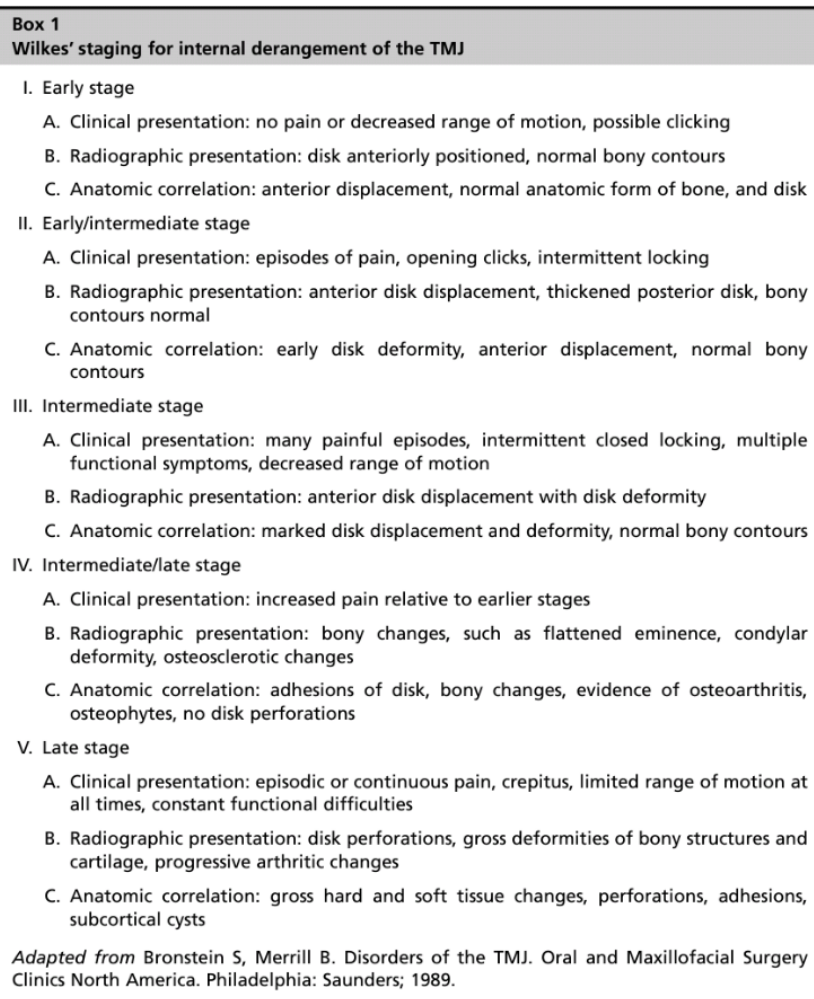

A. Clinical presentation: episodic or continuous pain, crepitus, limited range of motion at all times, constant functional difficulties

B. Radiographic presentation: disk perforations, gross deformities of bony structures and cartilage, progressive arthritic changes

C. Anatomic correlation: gross hard and soft tissue changes, perforations, adhesions, subcortical cysts

\section{Figure 2}

ment without reduction is a condition where the disc is displaced and acts as a definite obstruction between the condyle and eminence, this results in decreases mouth opening of the patient as the joint cannot translate forward, this is associated with intense pain as the condyle is resting on the retrodiscal tissue at the $12 \mathrm{o}$ clock position that is innervated with blood vessels and nerves that cause pain (Figure 3).

- Diagnosis of TMD includes a detailed case history of the patient, history of initiation of pain, exacerbating and relieving factors, any previous interventions done, any history of any dental procedure carried out for long hours.

- On examination the dentist/maxillofacial/clinician should palpate the joint by placing the index finger on the joint note any popping, clicking, crepitus sounds.

- Examiner should look out for any restriction in mouth opening, deviation of the mandible, tenderness in the muscles

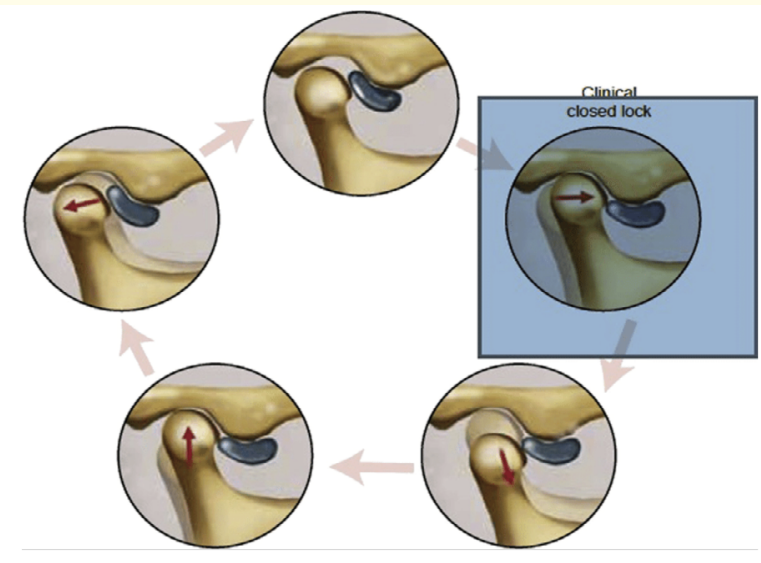

Figure 3

surrounding the joint, muscle spams, muscle dystonia (involuntary movement of a group of muscles), dental occlusion and dental causes of pain.

- For the purpose of radiological diagnosis earlier OPG (orthopantomograph) was done, but with advances MRI is considers as the first choice imaging modality for the temporomandibular joint.

Symptoms associated with TMJ:

- Jaw pain

- Headache

- Limitation of jaw opening

- Locking of jaw

- $\quad$ Earache

- Clicking/popping sounds

- Dull orofacial pain (capen art).

\section{Treatment}

The treatment of TMD is about improving the condition and standard of living of the patient and can be divided into two parts the conservative i.e. non-invasive methods and surgical i.e. invasive methods. Conservative treatment should be advised first followed by invasive therapies. Treatment in TMD should be aimed at pro- 
viding relief from pain, increase range of motion of the mandible, prevent further damage to the joint, improve the standard of living of a patient.

TMD is a multifactorial disorder hence the approach also has to be multifactorial. There are different modalities of treatment available for treating TMD and we will be discussing it as follows:

- Pharmacological treatment: Primarily while treating TMJ disorders the first line of drugs used are NSAIDS, Analgesics, Muscle relaxants, followed by corticosteroids, Opioids and Tricyclic anti depressants. Pharmacological treatment can be started for patients who have recently developed TMD symptoms and complain of muscle soreness and spasms. However, this line of treatment lacks merit because of the potential side effects of these drugs due to prolonged use to treat TMD, hence the need for diagnosing the root cause becomes imperative.

- Physical therapy: Physical therapy or jaw exercises have been considered to play a role in strengthening the masticatory muscles and improve the range of motion of the mandible in certain primary level cases in TMJ disorders. It involves gently massage over the affected muscle, guided opening and closing of mouth, different lateral movement exercises, TENS, IR etc.

- Splint therapy: Splint therapy has been used by maximum dentist since decades as a treatment for TMD, however it has been seen to have minimal effect on treating complex TMD cases. Splints are made of hard or soft clear acrylic that covers the mandibular and maxillary teeth.

The most commonly used splints in TMD are full arch stabilizing splint, followed by anterior repositioning splint and Posterior bite plain. The concept of using the splint is to unload the joint by changing the bite position and avoid the disc from getting sandwich between the condyle and the articular eminence, thereby providing time for the disc to heal. This concept is based on the healing and adapting capacity of the articular disc. Hard splint should be used on patients with parafunctional habits such as bruxism or clenching as these patients tend to bite through the splint if it is soft in consistency.

Occlusal adjustments, orthotics and neuromuscular dentistry: This is a form of treatment where the specialist uses various equip- ments to analyze the patients bite, muscle load, jaw movement analysis etc. We will understand in brief about a few:

I. Occlusal adjustments and orthotics: Tekscan is a digital occlusion analysis device that is capable of scanning the occlusal relationship of the teeth and maps the occlusal forces acting on the teeth while chewing with respect to time, thereby depicting the interferences, premature contacts and force imbalance in occlusion within almost $1 / 1000^{\text {th }}$ of a second.

II. Bioelectromyography (BioEmg, Tekscan): This is a device that measures the imbalance in the activity of muscles of head and neck by correlating it with interference in occlusion (Figure 4).

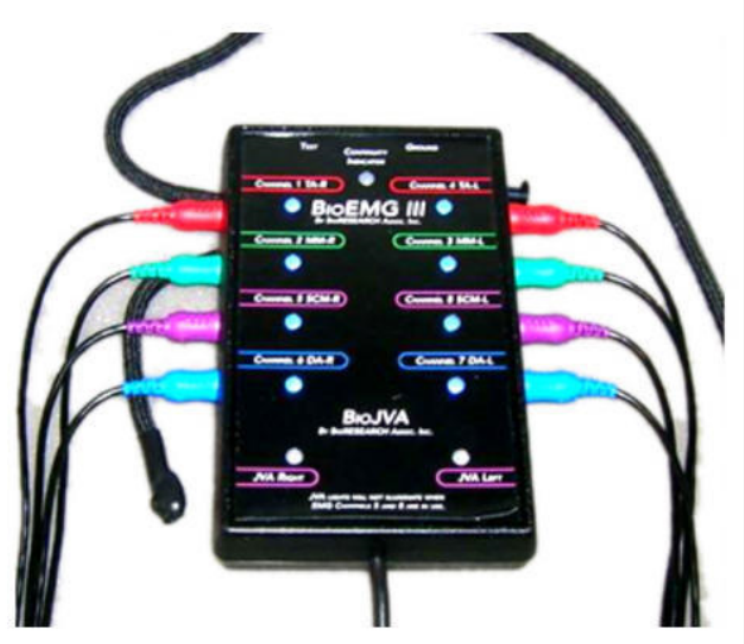

Figure 4

\section{Joint vibration analysis (JVA, Bioresearch associates):} It is a device that measures the condition of the joint with respect to its movements, disc structure and overall joint health. The vibrations emitted by this device helps the clinician to deduce a diagnosis (Figure 5).

IV. Botox injection: Botox injection (Botulinum Toxin A) in simple language is a toxin that blocks the uptake of the neurotransmitter acetylcholine from axon endings at the neu- 


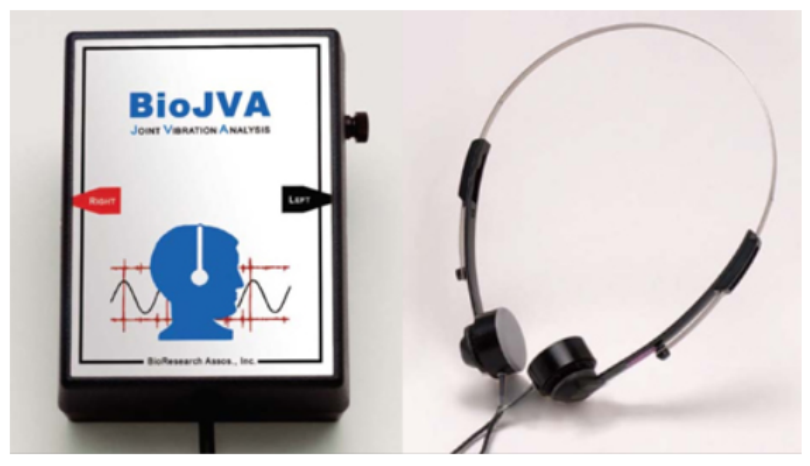

Figure 5

romuscular junction and thus causes muscle relaxation and reduces the hyperactivity of the muscle. Botox Injection in TMD is primarily used in cases of recurrent joint dislocation where the lateral pterygoid muscle is hyperactive or has spasms as the Lateral pterygoid muscle has a direct attachment at the anterior part of the disc and is responsible for the anterior translation of the disc, and recurrent dislocations in case of hyperactivity. Injecting the lateral pterygoid muscle with botox 25 units using an TMJ Arthroscope provides significant improvement in controlling the recurrent episodes of dislocations. Botox injections are also used in relieving spasms or Orofacial Dystonia in other muscles of mastication such as temporalis, masseter as it relieves the muscle of uncontrolled contractions. These treatment modules should be clubbed with an appropriate splint therapy to produce a synergistic effect in treatment of TMD.

Arthrocentesis: This is a procedure that bridges the gap between surgical and conservative TMJ treatment options. When all conservative methods of treating TMD are performed and remain unsuccessful for more than 6 months it is required to consider more invasive options depending on the clinical and radiological evidence. Arthrocentesis is a procedure that involves blind lavage of the joint with two18 gauze needle in the upper compartment of the joint using Ringer lactate (RL) solution and flushing of the inflammatory mediators present in the joint and can be performed under local anaesthesia, relieving the joint of the negative pressure thereby freeing the disc of any adherence to the bone. The rationale behind this procedure involves the flushing of these inflammatory mediators that are present in the joint because of disc interferences, mechanical obstruction, occlusal loading of the TMJ, that restrict the movement of the joint and cause pain, once these mediators are flushed from the joint it improves the range of motion and reduces pain significantly (Figure 6 and 7).

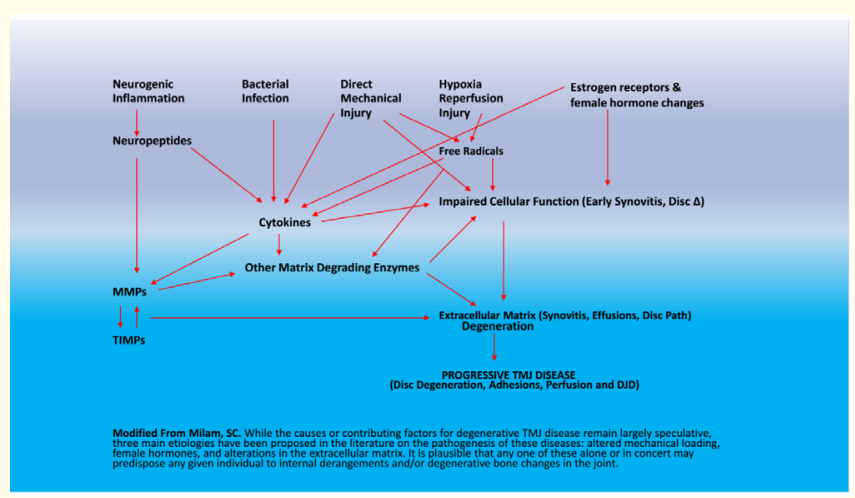

Figure 6

\begin{tabular}{|c|c|}
\hline \multicolumn{2}{|c|}{$\begin{array}{l}\text { Table } 6 \\
\text { Arthrocentesis for TMD treatment }\end{array}$} \\
\hline & Arthrocentesis \\
\hline Description & $\begin{array}{l}\text { Saline lavage of the superior joint space, hydraulic pressure and } \\
\text { manipulation to release adhesions, and elimination of intra-articular } \\
\text { inflammatory mediators (Fig. 3) } \text { (36,37; less invasive than arthroscopy and }^{\text {can be done in outpatient setting with local anesthesia and }} \\
\text { intravenous sedation }\end{array}$ \\
\hline Indication & $\begin{array}{l}\text { - Limited opening with anteriorly displaced articular disk without } \\
\text { reduction } \\
\text { - Chronic pain with good range of movement and displaced articular } \\
\text { disk with reduction } \\
\text { - Degenerative osteoarthritis }\end{array}$ \\
\hline Contraindications & $\begin{array}{l}\text { - TMJ with bony or fibrous ankylosis } \\
\text { - Extracapsular source of pain } \\
\text { - Patients who have not undergone noninvasive treatment modalities }\end{array}$ \\
\hline Efficacy & $\begin{array}{l}\text { Recently reported } 83.5 \% \text { treatment success rate in patients with internal } \\
\text { derangement and osteoarthritis (as defined as an improvement in } \\
\text { maximum jaw opening and a reduction in pain level and mandibular } \\
\text { dysfunction) }\end{array}$ \\
\hline
\end{tabular}

Data from Fonseca RJ. Oral and maxillofacial surgery. Chicago: Saunders; 2000.

\section{Figure 7}

Arthroscopy of TMJ: Arthroscopy of TMJ is the most recent advancement in the field of minimally invasive TMJ surgery. This procedure does not involve any incisions on the face and it can be 
performed under LA or GA, and is a safe and a precise procedure with minimal downtime for the patient. Arthroscopy is considered as a superior procedure to arthrocentesis and can be performed in two ways diagnostic arthroscopy or therapeutic arthroscopy; many TMJ surgeons have now started performing diagnostic arthroscopy substituting it for an MRI of the joint as the surgeon has a real time information about the joint and simultaneously can also perform a procedure if he/she wants. Therapeutic arthroscopy involves standard procedures that involve inspection, lavage, excision of fibrous bands and various disc repositioning procedures within the joint, a surgeon can also take biopsy of a suspicious structure inside the joint. Majority of the TMJ issues arise because of an anterior disc displacement in the TMJ, these disc repositioning procedure can be performed conveniently using arthroscopy.

Armamentarium for arthroscopy includes a $1.9 \mathrm{~mm}$ diameter scope which is either angled or straight, this scope uses the same landmarks as for arthrocentesis and enters the joint along with another port inserted from which a second instrument can be inserted to perform the surgery under constant RL irrigation. This assembly of instruments enables the surgeon to perform various procedures under direct vision on the screen and also maintain a record of findings and procedure.

\section{Open joint surgery}

TMJ open joint surgery may be indicated for the following:

A. Tumors

B. Disc perforations

C. Fibro Ankylosis

D. Bony ankylosis

E. Genetic/syndromic conditions

F. Condylar hypoplasia/hyperplasia

G. Hypermobility from neurological conditions

H. Total joint replacement surgery.

TMJ surgery is technically one of the more difficult surgical dissections in the maxillofacial region. While the close proximity of the facial nerve is the main reason for the difficult surgical access, other important anatomical structures such as the terminal branches of the external carotid artery and accompanying rich plexus of veins also add to the complexity of the dissection. With the middle cranial fossa above and the middle ear behind the TMJ, there is little room for surgical error as both these cavities are only a few millimetres away from the joint itself. That is why few oral and maxillofacial surgeons have the inclination, confidence, skill and experience to tackle such a small inaccessible joint, which is approached via an incision in the skin crease in front of the ear.

There are a lot of procedures such as eminectomy or arthrotomy that involve removal or shaving of the eminence releasing the pressure on the disc but there is lack of long term evidence on this particular procedure.

Discectomy has become the mainstay treatment in treating internal derangement as it has a follow up of $>20$ years, it involves the removal of the entire disc. Earlier after discectomy they would leave the outcome on the remodelling capacity of the disc and condyle, which would lead to osteoarthritis, with research it became evident that the disc should be replaced and the best options to consider for it were autogenous fat graft, conchal cartilage, temporalis fascia, dermis graft from lower abdomen.

Total joint replacement is considered for the end stage TMJ disease such as severe trauma, tumor where no components of the TMJ can be salvaged and both the disc as well as the condyle has to be removed. This leaves the patient with a facial asymmetry and malocclusion which then has to be treated with a alloplastic or a autogenous joints such as costochondral graft etc. It addresses the joint functionality and any pain relief obtained is additional component of the treatment (Figure 8) [1-6].

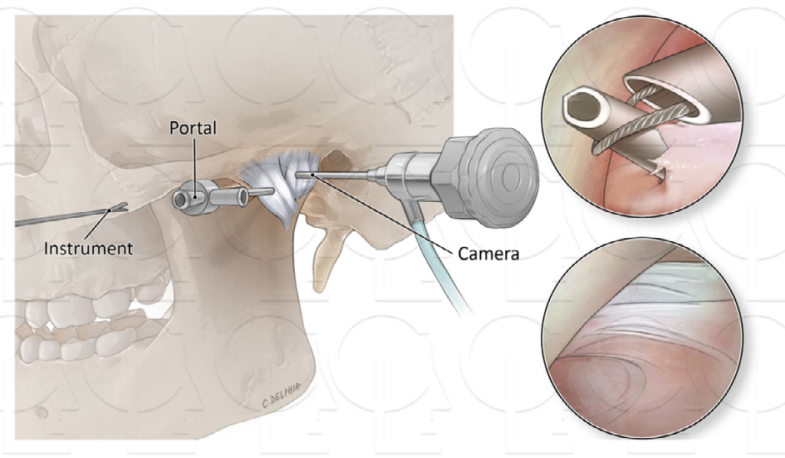

Figure 8 


\section{Conclusion}

The general dental team has a role to play in the diagnosis, conservative management and referral of patients with TMJ pain. By understanding the various causes and presentations of TMJ problems it is possible to distinguish between dental pain, TMJ disorder and the more complicated facial pains to ensure speedy diagnosis and management for our patients. TMJ disorders are a common disorder that represents with a lot of pain and discomfort, it may not be a life threatening disorder but it is surely a life altering one. It is of paramount importance to have an understanding about conservative treatment and surgical treatment protocol and a guideline to follow for all dentist and maxillofacial surgeons to diagnose and treat a TMJ. Each patient should undergo a complete history and examination followed by a comprehensive plan of management (Figure 9-12).

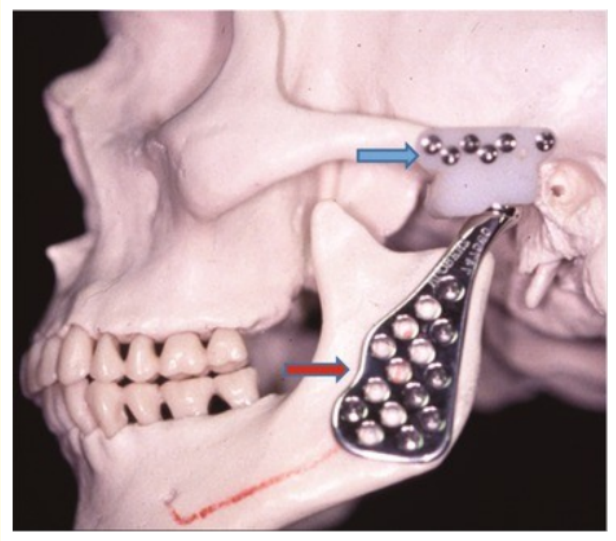

Figure 9

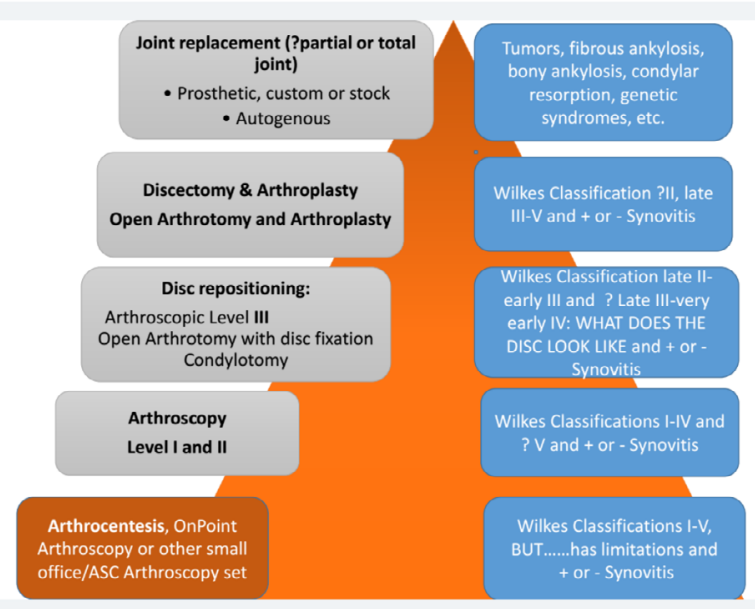

Figure 10

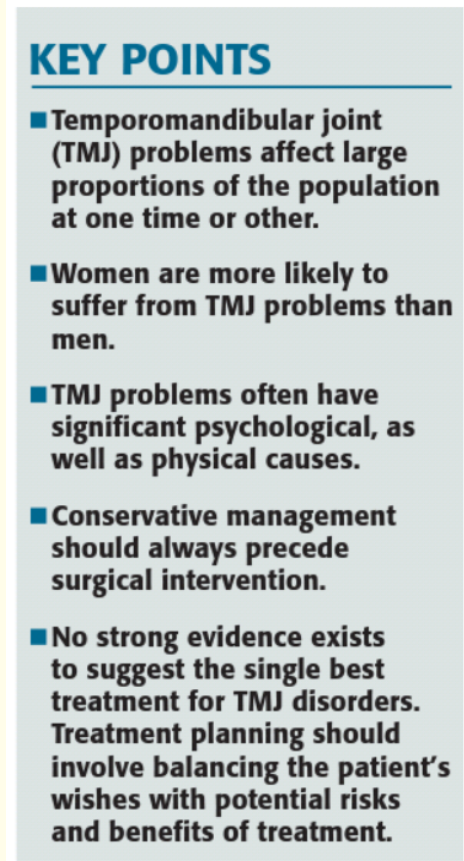

Figure 11

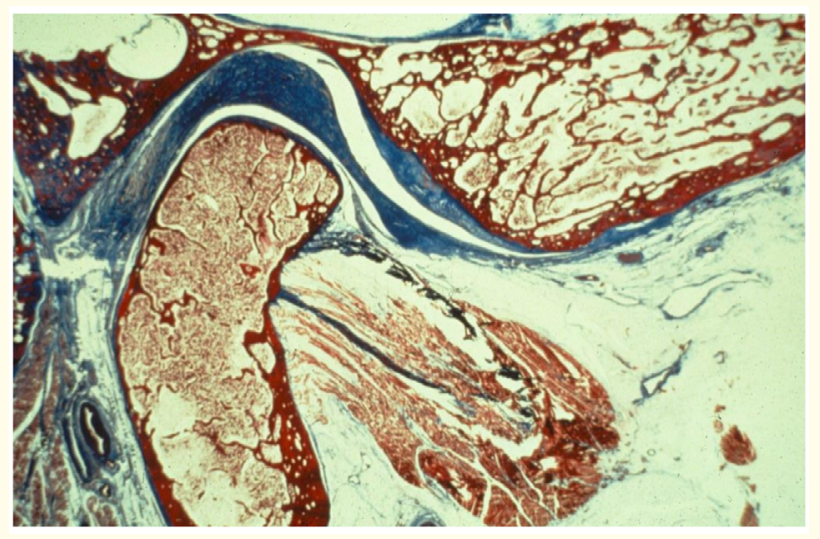

Figure 12

\section{Bibliography}

1. Berkovitz B., et al. Master Dentistry. Oral biology. Churchill Livingstone Elsevier, Edinburgh 3 (2011).

2. Ghali GE., et al. "Peterson's principles of oral and maxillofacial surgery". $3^{\text {rd }}$ edition. Shelton (CT): Pmph USA (2012).

3. "Diagnosis, treatment and management of TMJ disorders". Dental Nursing (2011). 
4. Abubaker AO., et al. "Estrogen and progesterone receptors in temporomandibular joint discs of symptomatic and asymptomatic persons: a preliminary study". Journal of Oral and Maxillofacial Surgery 51 (1993): 1096-100.

5. Mohlin B and Kopp S. "A clinical study on the relationship between malocclusion, occlusal interferences, and mandibular pain and dysfunction". Swedish Dental Journal 2 (1978): 103.

6. Thaddeus Connelly., et al. "Contemporary Management of Temporomandibular Disorders".

Volume 5 Issue 7 July 2021

(C) All rights are reserved by Tofiq Hakimuddin Bohra., et al. 